Proceedings of the Symposium K: "Complex Oxide Materials for New Technologies" of E-MRS Fall Meeting 2006, Warsaw, September 4-8, 2006

\title{
Magneto-Optics of Spontaneous and Field Induced Vortices in Twinned $\mathrm{YBa}_{2} \mathrm{Cu}_{3} \mathrm{O}_{7-\delta} / \mathrm{La}_{1-x} \mathrm{Sr}_{x} \mathrm{MnO}_{3}$ Bilayers
}

\author{
R. Gerbaldo, G. Ghigo, L. Gozzelino, F. Laviano, \\ E. Mezzetti, B. Minetti \\ Department of Physics, Politecnico di Torino \\ c.so Duca degli Abruzzi 24, 10128 Torino, Italy and \\ INFN Sez. Torino, Via P. Giuria 1, 10125 Torino, Italy \\ P. Przysłupski, A. Tsarou and A. Wiśniewski \\ Institute of Physics, Polish Academy of Sciences \\ al. Lotników 32/46, 02-668 Warszawa, Poland
}

The electromagnetic coupling between the magnetic and the superconducting layers in perovskite heterostructures is investigated by means of the magneto-optical technique. The quantitative imaging of the magnetic field distribution allows the high-resolution reconstruction of local supercurrent density. Two phenomena arising from the coupling between manganite layer and the $\mathrm{YBa}_{2} \mathrm{Cu}_{3} \mathrm{O}_{7-\delta}$ superconducting film deposited on top are addressed, i.e., the local effects of the electronic coupling between the compounds and the interaction between the manganite magnetic moments and the vortices in the superconductor. The first issue can be quantified in terms of the local superfluid density depression/enhancement in correspondence to the underlying magnetic structure, both domains and domain walls (in dependence on the orientation and sign of the local magnetization) and of the spontaneous, macroscopic screening current loops generated by the manganite domain walls. The interaction between the local manganite magnetization and the superconducting vortices is also influenced by structural defects because they modulate the magnetic pattern of the manganite. Different channelling phenomena in correspondence to the natural twin boundaries of the substrate, but locally magnetized by pinned domain walls of the manganite on their locations, are recognized.

PACS numbers: 74.25.Ha, 74.25.Qt, 74.78.Db, 74.78.Fk

\section{Introduction}

Superconducting/ferromagnetic (SC/FM) systems made with transition metal compounds display either unexplained or new phenomena of current solid state physics [1]. In particular, the study of interplay between two competing or- 
der parameters that interact but do not cancel themselves is especially interesting in all-perovskite heterostructures (generally cuprate films are well grown on manganite layers), because of the strongly correlated electronic ground states in both magnetic and superconducting compounds [2].

In an SC/FM perovskite bilayer with single layers thicker than a few unit cells, the magnetic interaction should dominate the electrodynamical response of the system. In a recent theoretical proposal, Bulaevskii et al. [3] predicted that the magnetic interaction could cause strong pinning of the magnetic structure of the superconducting vortices by the striped magnetic pattern of the FM layer, where domains are alternating on the length scale of the London penetration depth with opposite out-of-plane magnetization. Previous measurements indeed found an average increase in the vortex pinning in SC/FM bilayers $[4,5]$, although the details of magnetic pattern were unknown. Recent experiments on decoupled SC/FM heterostructures confirmed that the average increase in the vortex pinning should come only from the magnetic interaction between the layers [6]. It is worth noting that we should expect a rich phenomenology in dependence on the details of the magnetic pattern, especially in such magnetic oxides with a strongly correlated electronic ground state where many different domain structures are expected [7]. The interaction between a magnetic domain wall (DW) and a vortex was studied by Helseth et al. [8] and, in fact, they found both attractive and repulsive forces in dependence on the alignment between the DW magnetization and the magnetic field of the vortex. Moreover, for magnetic layers with out-of-plane magnetization, it has also been proposed that a new topological texturing should spontaneously occur in both layers [9].

In order to observe the local interaction between the FM microstructures and the SC flux quanta in the bilayer, a high resolution magnetic imaging technique becomes a necessary tool. In a previous work, we used the magneto-optical (MO) imaging technique to show directly that vortices in $\mathrm{YBa}_{2} \mathrm{Cu}_{3} \mathrm{O}_{7-\delta}$ (YBCO) on $\mathrm{La}_{0.67} \mathrm{Sr}_{0.33} \mathrm{MnO}_{3}$ (LSMO) bilayers are spontaneously generated and then they are either attracted or repulsed by coherent out-of-plane DWs induced in the manganite due to the twinned substrate [10]. The splitting of the magnetic pattern due to twin boundaries (TBs) in a bilayer with $\mathrm{La}_{0.885} \mathrm{Sr}_{0.115} \mathrm{MnO}_{3}$ allows observing the vortex diffusion also inside extended out-of-plane domains [11].

In this paper, we consider the contribution of the magnetic interaction to the vortex nucleation and pinning in such twinned SC/FM bilayers. It turns out that the magnetic interaction we observed between vortices and localized magnetic moments in the manganite is either enhancing or depressing the local vortex pinning in dependence on the magnetization orientation of the domain (or DW), with respect to the direction of the applied field.

\section{Experimental details}

All the bilayers here presented were grown on twinned single crystals of $\mathrm{LaAlO}_{3}$, by multi-target high-pressure dc sputtering from the corresponding sto- 
ichiometric targets $[12,13]$. The manganite layers of LSMO, with doping levels $x=0.33$ and $x=0.115$, are $100 \mathrm{~nm}$ thick and on top of them a $50 \mathrm{~nm}$ thick YBCO film was grown.

The $\mathrm{LaAlO}_{3}$ substrate has a large number of coherent TBs, mostly organized in parallel families or displaying characteristic ripples (zig-zag structure), see the optical image in Fig. 1a [14].
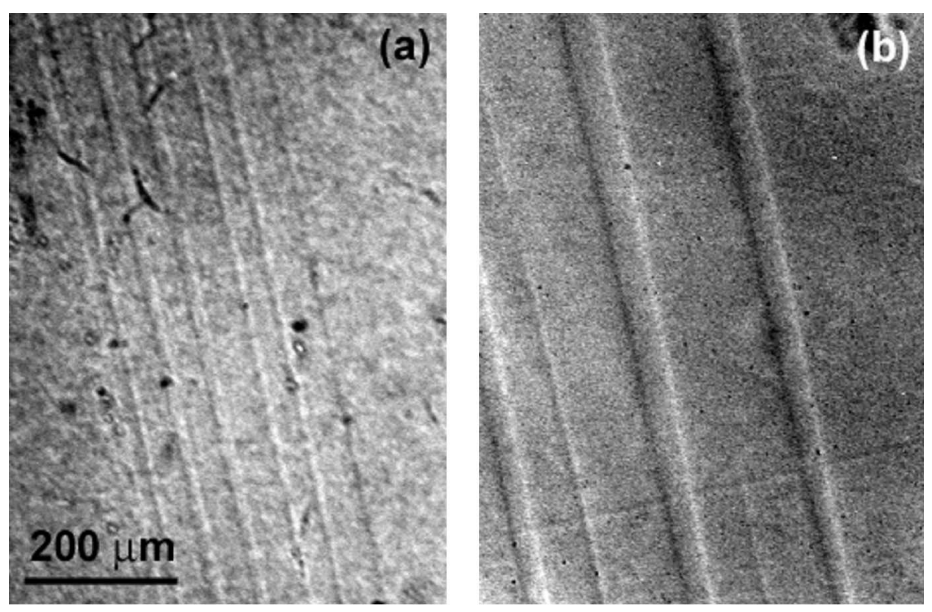

Fig. 1. (a) Optical image of the bilayer with the metallic LSMO. (b) MO image of the very same region of the sample taken at $T=100 \mathrm{~K}$. Bright and dark straight lines in the magnetic pattern indicate the presence of out-of-plane magnetic moments pinned at a subset of the TB family (bright lines mean magnetic moment tilted up with respect to the film plane, dark lines mean magnetic moment with the opposite direction).

The MO imaging of the magnetic distribution generated by flat materials is achieved by the use of a Bi-doped iron garnet film, with in-plane magnetization, as the MO indicator [15]. The magnetic imaging is performed in a reflection microscope equipped with Glan-Thompson polarizers, Hg arc lamp, and high resolution digital camera [16]. The indicator thickness and the finite distance from the magnetic source are the limiting factors for the spatial resolution, in addition to the light wavelength limit. To achieve the best spatial resolution, we have chosen an indicator thickness of less than 1 micron with very thin mirror layer (less than $20 \mathrm{~nm}$ ) and then the indicator was tightly pressed on top of the cleaned bilayer surface. In this way, we obtain a spatial resolution near to the limit of the used light wavelength of the order of $500 \mathrm{~nm}$.

The quantitative evaluation of the supercurrent distribution is achieved by means of a nonlinear calibration of the magnetic field outside the sample area [17]. For this purpose, the YBCO film of the bilayer (with insulating LSMO) was patterned by UV photolithography and chemical wet etching in a $\mathrm{HCl} / \mathrm{H}_{2} \mathrm{O}(0.37 \%)$ solution. 
Moreover, since we are dealing with a SC/FM bilayer that both contribute to the detected magnetic moment distribution, we must apply a special procedure in two steps, i.e., generation of a stable magnetic pattern in the manganite above the SC $T_{\mathrm{C}}$ by means of an in-plane magnetic field, the former, and subtracting the low temperature magnetic field distribution, the latter. Then, applying a sequence of increasing out-of-plane magnetic fields, we obtain only the contribution of the SC layer to the measured magnetic moment, i.e., screening currents and vortices generated by the external out-of-plane field.

\section{Results}

The magnetic pattern of the bilayer with the metallic LSMO contains out-of-plane magnetic moments, even at room temperature because of its high transition temperature $\left(T_{\text {Curie }} \approx 360 \mathrm{~K}\right)$, as also shown in Fig. 1 b. These nonzero out-of-plane magnetic moments (bright and dark stripes) are localized at subsets of the TB family (the localization of these magnetic structures only at TBs was positively verified by comparison with the optical image in the same zone for any sample displaying TBs in the substrate). The splitting of the magnetic domains is expected in the presence of TBs because they are good pinning centers for DWs, whose coercivity is strongly enhanced by TBs [18]. Also previous magneto-optical imaging of $\mathrm{La}_{0.67} \mathrm{Ca}_{0.33} \mathrm{MnO}_{3}$ manganite films, deposited by pulse laser deposition on $\mathrm{SrTiO}_{3}$ substrates [19], demonstrated that the twin boundaries, generated in the substrate by a martensitic transition, tilt out-of-plane the local magnetic moments of the manganite.

Thus, the twinned bilayer with the metallic LSMO displays in-plane domains that are separated by out-of-plane DWs strongly pinned at TBs. Such magnetic pattern with out-of-plane magnetic moments displayed at room temperature remains unchanged below the $\mathrm{SC} T_{\mathrm{C}}$ (see Fig. 2a). Therefore, the local magnetic flux threading the SC film is shrunk in the vortex cores and the magnetic structure of the vortices smoothly decorates all the underlying magnetic pattern.

Then vortices generated by the application of an out-of-plane uniform external field are going to interact with vortices spontaneously nucleated and pinned at TBs, with the DWs also pinned at TBs and with the TBs themselves. It was already pointed out that the pinning of such magnetized TBs is very different from the behaviour observed in twinned YBCO films and crystals [20]. From Fig. 2b, it turns out that vortices pile up on the DWs with the magnetization aligned along the external field and a vortex-antivortex annihilation on DWs of the opposite sign.

More interestingly, when the TBs are parallel to the Lorentz force exerted by the screening currents on vortices, a guided channelling of the vortices is observed [10]. This guided motion is modulated by changing the magnetization state of DWs, i.e., vortices aligned parallel to the magnetization of the DWs proceed further into the sample with respect to those aligned antiparallel. The vortex 


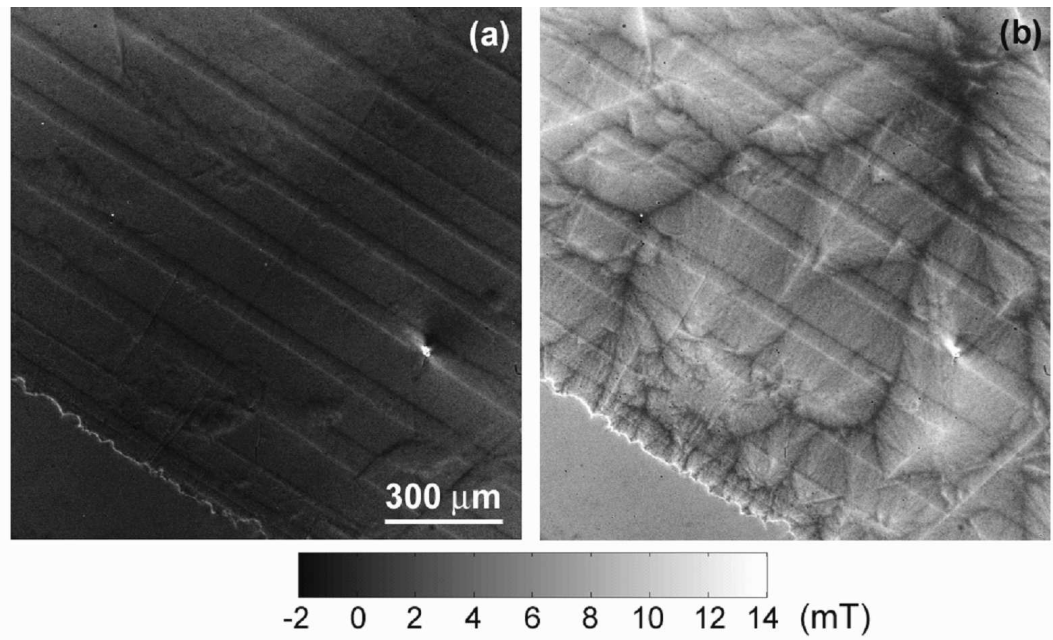

Fig. 2. (a) Magnetic field distribution after ZFC of the bilayer with the metallic LSMO at $T=4.2 \mathrm{~K}$. On the bottom-left part of the frame, the sample edge is visible. The DW pattern is frozen as superconducting vortices. Grey areas in between DWs are flux-free. (b) Magnetic field distribution at $T=4.2 \mathrm{~K}$ and $\mu_{0} H_{\text {app }}=6.1 \mathrm{mT}$.

channelling occurs in correspondence of the TBs, in the part of the critical state profile at the border with the flux-free zone, see Fig. 3.

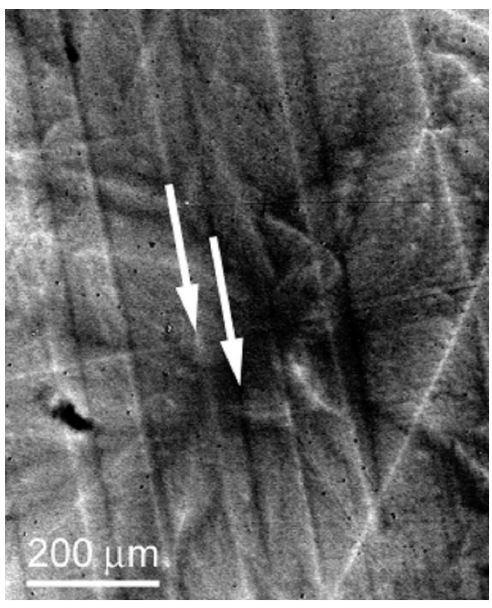

Fig. 3. MO image of the central part in the bilayer with the metallic LSMO. Vortices diffuse towards the sample center, but it is clear that channeling through TBs occurs in dependence on the local magnetic moment orientation with respect to the external field. The arrows point to TBs with opposite magnetizations: those TBs displaying counter-oriented magnetization with respect to the applied field (dark straight lines) are able to block incoming vortices. 
On the contrary, for the bilayer with the insulating LSMO, we observed a stable magnetic pattern characterized by alternating out-of-plane domains. Such a pattern was induced in the bilayer with the insulating LSMO by means of a polarization in-plane field larger than the saturation field above the $\mathrm{SC} T_{\mathrm{C}}$ (at $T=100 \mathrm{~K})$.

As explained in the experimental details, the YBCO film was etched in a disk shape of $200 \mu \mathrm{m}$ diameter in order to measure all the internal and external stray field generated by the supercurrent flowing in the sample. The striped magnetic field distribution of the manganite layer, shown in Fig. 4a, is frozen when the sample is cooled below the YBCO $T_{\mathrm{C}}$, like the above discussed sample. The spontaneously induced vortex distribution is much more uniform with respect to
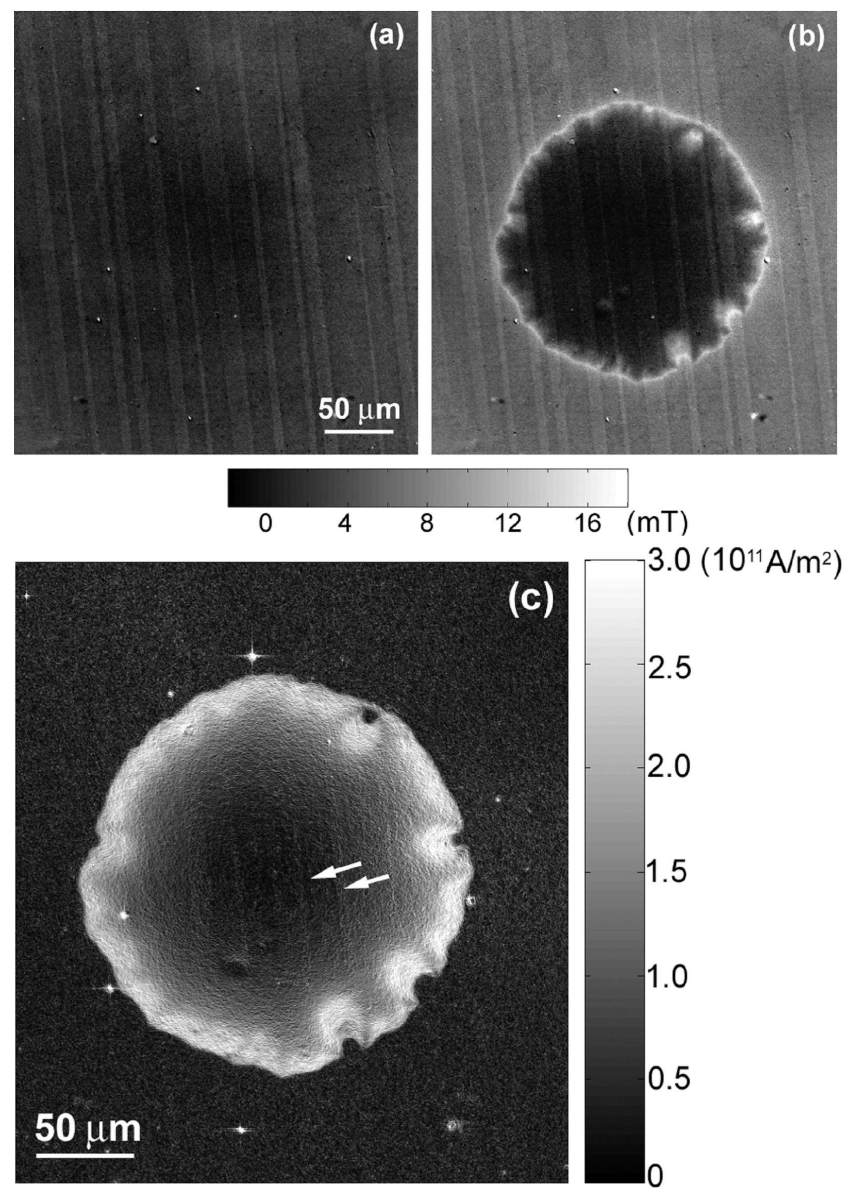

Fig. 4. Magnetic field distribution of the bilayer with the insulating LSMO at $T=4.2 \mathrm{~K}$ (a) just after the nominal ZFC and (b) under an out-of-plane field of $\mu_{0} H_{\text {app }}=4.0 \mathrm{mT}$. (c) Supercurrent density modulus distribution at $T=4.2 \mathrm{~K}$ and $\mu_{0} H_{\text {app }}=4.0 \mathrm{mT}$. Arrows indicate two adjacent counter-flowing loops in the central shielded region of the SC sample. 
the previous sample, because the vortices are decorating large domain areas instead of being confined in DWs. After applying an uniform external magnetic field perpendicular to the bilayer, other vortices start to be nucleated at the sample edges, while the spontaneous vortices stay pinned, see Fig. $4 \mathrm{~b}$.

The quantitative measurement of the supercurrent density, whose modulus distribution is reported in Fig. 4c, reveals that the central supercurrent loop, inside the area of the sample not yet reached by the vortices coming from the edges, is also split in different domains. The loops establish in correspondence of alternating domains, with different sign of the magnetization, and we found that adjacent domains with opposite magnetization direction trigger counter-flowing loops. This supercurrent distribution is very similar to the supercurrent pattern predicted by Erdin et al. [9], though here that magnetic pattern is fixed by the TBs.

The modulation of the critical current in the zone near the edges is quite smooth and we measured a slight modulation of the order of $15 \%$ [11]. This order of magnitude for the magnetic pinning seems in contradiction with the theory on magnetic pinning [3], but here we are dealing with alternating magnetic domains that have an average width of $50 \mu \mathrm{m}$, by far larger than the mean inter-vortex spacing. Moreover, we note that strong modulations of the critical state profile occur, either enhancing or depleting the vortex diffusion inside the sample, see Fig. 5. The flux profile modulation is isotropic with respect to the vortex diffusion, i.e., for vortices moving parallel (as indicated by the arrows in Fig. 5) or

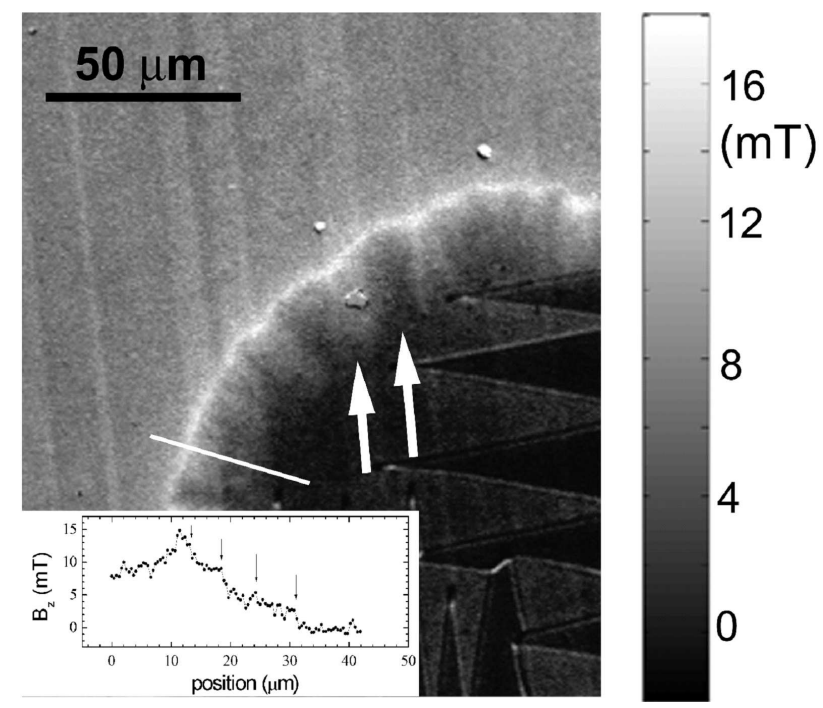

Fig. 5. Magnetic field distribution at $T=4.2 \mathrm{~K}$ and $\mu_{0} H_{\text {app }}=5.9 \mathrm{mT}$ in the bilayer with insulating LSMO. The arrows indicate different critical state fronts diffusing in two contiguous domains with opposite magnetization. In the inset, the line profile across the white line in the magnetic field map demonstrates the modulation of the flux density induced by the underlying magnetization of the manganite domains. 
perpendicular to the alternating domain arrangement, see the inset of Fig. 5. We ascribe this modulation to the presence of spontaneous vortices that are nucleated by the domain stray field when cooling below the YBCO $T_{\mathrm{C}}$, as discussed above.

\section{Conclusions}

The magnetic field imaging of twinned SC/FM perovskite bilayers results to be useful for studying both the magnetic behaviour of the single layers and their mutual interaction. We found that the nonzero magnetic flux generated by the FM layer is frozen in the SC film when the sample is cooled below the SC $T_{\mathrm{C}}$. The resulting vortex pattern decorates the magnetic structure (in this case fixed by the DWs pinning at TBs). The intrinsic pinning of TBs on vortices is modulated by the localized magnetic moments in the FM layer in such a way that vortices channelling along the TBs can be pushed ahead or backward in dependence on the orientation of the DW magnetization with respect to the external field that generates the vortices.

When the sample displays out-of-plane magnetic domain and in-plane DWs, we found a modulation of the critical current (15\%) and a novel supercurrent pattern in the shielded region. These findings are consequence of the spontaneous vortex nucleation occurring in the SC/FM bilayer when the FM layer shows a multi-domain pattern on the micron scale and $T_{\text {Curie }}>T_{\mathrm{C}}$.

\section{Acknowledgments}

Work partially supported by the Programme of Scientific and Technological Co-operation between the Italian Republic and the Republic of Poland 2004-2006. The work was also supported by the Polish Ministry of Science and Higher Education under project for the years 2006-2009 (MNiSW-1 P03B 122 30). The Italian partners acknowledge the contribution of the MIUR-PRIN project no. 2004037901.

\section{References}

[1] A.I. Buzdin, Rev. Mod. Phys. 77, 935 (2006).

[2] J. Chakhalian, J.W. Freeland, G. Srajer, J. Strempfer, G. Khaliullin, J.C. Cezar, T. Charlton, R. Dalgliesh, C. Bernhard, G. Cristiani, H.-U. Habermeier, B. Keimer, Nature Phys. 2, 244 (2006).

[3] L.N. Bulaevskii, E.M. Chudnovski, M.P. Maley, Appl. Phys. Lett. 76, 2594 (2000).

[4] A. Garcia-Santiag, F. Sanchez, M. Varela, J. Tejada, Appl. Phys. Lett. 77, 2900 (2000).

[5] H.-U. Habermeier, J. Albrecht, S. Soltan, Supercond. Sci. Technol. 17, S140 (2004).

[6] J. Albrecht, S. Soltan, H.-U. Habermeier, Phys. Rev. B 72, 092502 (2005).

[7] A. Hubert, R. Schafer, Magnetic Domains: The Analysis of Magnetic Microstructure, Springer-Verlag, Berlin 1998. 
[8] L.E. Helseth, P.E. Goa, H. Hauglin, M. Baziljevich, T.H. Johansen, Phys. Rev. $B$ 65, 132514 (2002).

[9] S. Erdin, I.F. Lyuksyutov, V.L. Pokrovsky, V.M. Vinokur, Phys. Rev. Lett. 7, 017001 (2002); R. Laiho, E. Lahderanta, E.B. Sonin, K.B. Traito, Phys. Rev. B 67, $144522(2003)$.

[10] F. Laviano, L. Gozzelino, E. Mezzetti, P. Przyslupski, A. Tsarev, A. Wisniewski, Appl. Phys. Lett. 86, 1644 (2005).

[11] L. Gozzelino, F. Laviano, P. Przyslupski, A. Tsarou, A. Wisniewski, D. Botta, R. Gerbaldo, G. Ghigo, Supercond. Sci. Technol. 19, S50 (2006).

[12] P. Przyslupski, A.Wisniewski, R. Szymczak, J. Igalson, Czech. J. Phys. 46, 1355 (1996); P. Przyslupski, S. Kolesnik, E. Dynowska, T. Skoskiewicz, M. Sawicki, IEEE Trans. Appl. Supercond. 7, 2192 (1997).

[13] P. Przyslupski, A. Tsarou, P. Dluzewski, W. Paszkowicz, R. Minikayev, K. Dybko, M. Sawicki, B. Dabrowski, C. Kimaball, Supercond. Sci. Technol. 19, S38 (2006).

[14] E.B. McDaniel, J.W.P. Hsu, J. Appl. Phys. 80, 1085 (1996).

[15] Ch. Jooss, J. Albrecht, H. Kuhn, S. Leonhardt, H. Kronmüller, Rep. Prog. Phys. 65, $651(2002)$

[16] F. Laviano, D. Botta, A. Chiodoni, R. Gerbaldo, G. Ghigo, L. Gozzelino, S. Zannella, E. Mezzetti, Supercond. Sci. Technol. 16, 71 (2003).

[17] Ch. Jooss, A. Forkl, R. Warthmann, H.-U. Habermeier, B. Leibold, H. Kronmüller, Physica C 266, 235 (1996).

[18] A.J. Kurtzig, IEEE Trans. Magn. 6, 497 (1970).

[19] V. Vlasko-Vlasov, Y.K. Lin, D.J. Miller, U. Welp, G.W. Crabtree, V.I. Nikitenko, Phys. Rev. Lett. 84, 2239 (2000).

[20] V.K. Vlasko-Vlasov, L.A. Dorosinskii, A.A. Polyanskii, V.I. Nikitenko, U. Welp, B.W. Veal, G.W. Crabtree, Phys. Rev. Lett. 72, 3246 (1994); C.A. Durán, P.L. Gammel, D.J. Bishop, J.P. Rice, D.M. Ginsberg, Phys. Rev. Lett. 74, 3712 (1995); U. Welp, T. Gardiner, D.O. Gunter, B.W. Veal, G.W. Crabtree, V.K. Vlasko-Vlasov, V.I. Nikitenko, Phys. Rev. Lett. 74, 3713 (1995). 